\title{
Coulomb Blockade of a Three-terminal Quantum Dot
}

\author{
Robert Andrzej Żak ${ }^{1,2}$ and Karsten Flensberg ${ }^{2}$ \\ ${ }^{1}$ Institute of Theoretical Physics, Faculty of Physics, \\ University of Warsaw, ul. Hoża 69, PL-00-681 Warsaw, Poland \\ ${ }^{2}$ Nano-Science Center, Niels Bohr Institute, University of Copenhagen, \\ Universitetsparken 5, DK-2100 Copenhagen Ø, Denmark
}

\begin{abstract}
We study an interacting single-level quantum dot weakly coupled to three electrodes. When two electrodes are biased by voltages with opposite polarities, while keeping the third lead (the stem) grounded, the current through the stem is a measure of electron-hole asymmetry of the dot. In this setup we calculate the stem current for both metallic and ferromagnetic (collinearly polarized) leads and discuss how the three-terminal device gives additional information compared to the usual twoterminal setup. We calculate both the sequential and cotunneling contribution for the currents. For the latter part we include a regularization procedure for the cotunneling current, which enables us to also describe the behavior at the charge degeneracy points.
\end{abstract}

\section{INTRODUCTION}

A systems consisting of a quantum dot weakly coupled to external leads have been extensively studied, both for unpolarized $1,2,3$ and polarized leads,,$\underline{4,5,6,7,8,9}$ These all dealt with two-terminal transport properties, with a third electrode acting as a capacitively connected gate.

System with more than two terminals has a long history in mesoscopic physics $\underline{10}$ The functionality added by the third lead is of importance for some applications, in particular the so-called Y-branch structures: $11,12,13,14$ These studies consider open mesoscopic systems, where correlation effects can be neglected. Some recent works have studied the opposite case where correlations are important, e.g. the study of current-current correlations in a three-terminal device, $\stackrel{15,16}{16}$ Kondo peaks in a three/four-terminal setup, $\stackrel{17,18}{\underline{1}}$ and crossed carbon nanotubes $\frac{19,20,21}{1}$ Here, we consider a multi-terminal quantum dot and, in particular, the non-linear response, which brings in new information about the structure of the quantum dot as well as about the magnetization of side branches.

The system we study in detail consists of a single level quantum dot coupled to three leads, Fig. 1, We allow collinear spin polarization of leads, so that some of them can be polarized while others are kept unpolarized. For the sake of simplicity, the polarization, if any, is assumed to be complete. To lift the spin degeneracy, we apply a magnetic field to the dot collinear with polarization of the leads. We study the current through the central junction (referred to as a stem) and its dependence on the applied voltage, magnetic field, position of dot's energy states, and orientation of leads' polarization. Except for the limiting case of non-interacting electrons, the exact solution is not known, and approximative methods are widely applied. In the typical experimental setup a coupling between the dot and the lead is of order of $\mu \mathrm{eV}$ while temperature of order of $\mathrm{meV}$ or higher, therefore it is often justified to perform perturbation expansion in a small parameter $\Gamma / k_{B} T$. We cut off the perturbation expansion on second order.

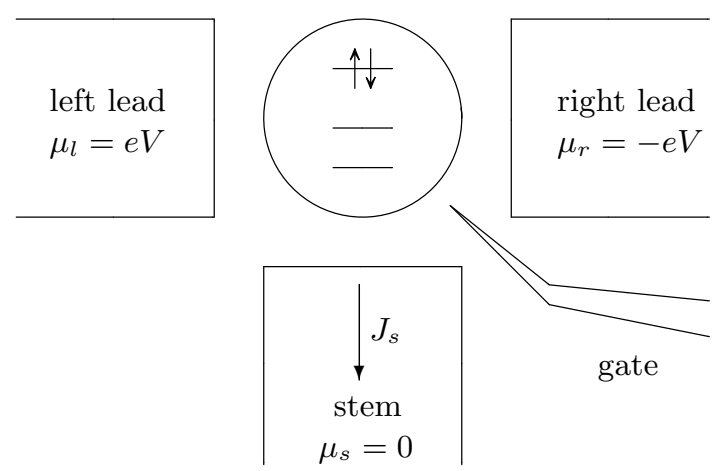

FIG. 1: The three terminal device. The occupation number is controlled via the gate by applying the voltage $V_{g}$. The stem chemical potential, $\mu_{s}=0$, serves as the reference level. The left and right leads are biased in push-pull manner with $\mu_{l}=e V$ and $\mu_{r}=-e V$.

In first order, we derive the sequential tunneling current through a central electrode, Eq. (9). When the system is biased symmetrically, $V_{l}=V$ and $V_{r}=-V$, the stem current is an even function of applied voltage and an odd function of the gate voltage, Fig. 4. The stem current can be either negative or positive depending on the nature of the electron transport.

To understand this, we first observe that the current from the left to the right lead occurs at low temperatures via only two charge states and can be predominately either electron- or hole-like. If the energy difference between the $\nu+1$ and $\nu$ electron states is positive, the transport is electron-like and hole-like if it is negative, with $\nu$ standing for the occupation number. For the electron-like case the $\nu+1$ state can decay via an electron leaving through the stem, whereas for the hole-like case, the $\nu$ state can decay by the electron entering from the stem. Hence the stem current is zero for the electron-hole symmetric case.

The three terminal setup therefore measures the electron-hole asymmetry, and in this respect it is similar to thermopower $\stackrel{22}{2}$ Interestingly, a change between the two situations above, can be induced by the magnetic 


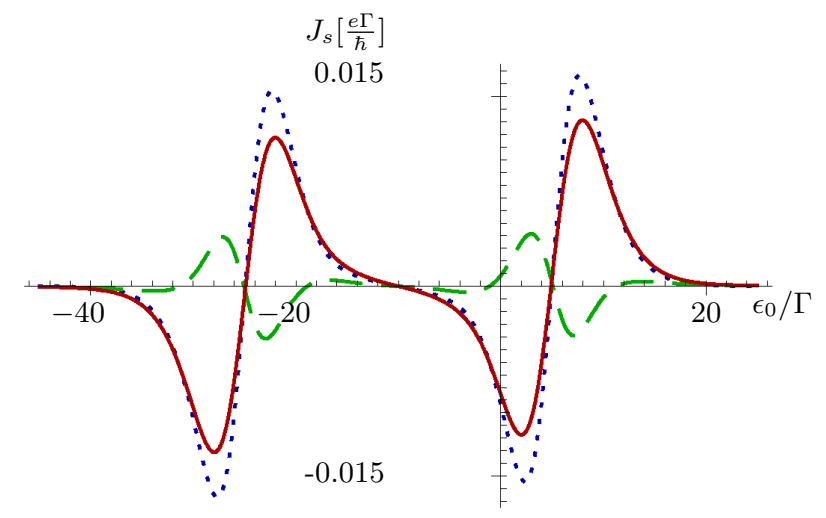

FIG. 2: (Color online) The stem current (solid, red) being the sum of the sequential part (dotted, blue) and the cotunneling (dashed, green). The sequential contribution gives sufficient qualitative description. For this plot leads are unpolarized with $\Gamma_{l}=\Gamma_{r}=\Gamma_{s}=\Gamma / 3, U=20 \Gamma, B=5 \Gamma, k_{B} T=2 \Gamma$ and $V / \Gamma=1$.

field applied to the dot which modifies the physics significantly, see Fig. 5. Moreover, in a three-terminal setup with magnetized side branches and the non-magnetic stem the current value enables one to distinguish among four magnetization alignments, Fig. 6.

In second order, the total current is increased by virtual cotunneling processes for these values of the gate voltage for which the sequential tunneling is exponentially suppressed. Furthermore, the cotunneling correction lowers the sequential current maxima and gives rise to additional broadening of order of the sum of all couplings $\Gamma_{s}+\Gamma_{l}+\Gamma_{r}$, and hence the total broadening is the sum of the thermal part and the latter. However, we will show that the cotunneling current does not change the sensitivity towards electron-hole asymmetry, and therefore the qualitative picture described above still holds to higher order in tunneling, see Fig. 2 ,

In the Appendix the exact solution for non-interacting electrons is compared with the perturbative treatment and even though the renormalization of level positions also contributing to the second order current was neglected, we achieve good agreement between the two.

\section{MODEL HAMILTONIAN}

The model Hamiltonian of the quantum dot connected to any number of unpolarized leads is

$$
H=H_{L}+H_{T}+H_{D}
$$

where

$$
H_{L}=\sum_{j} H_{L}^{j}=\sum_{j} \sum_{k \sigma} \xi_{j, k \sigma} c_{j, k \sigma}^{\dagger} c_{j, k \sigma}
$$

describes the uncoupled leads indexed by $j$ while $c_{j, k \sigma}^{\dagger}$ and $c_{j, k \sigma}$ form an orthogonal set of creation and annihi- lation operators in lead $j$.

The dot is described by the Hamiltonian

$$
H_{D}=\sum_{\sigma}\left(\epsilon_{0}-\sigma B\right) d_{\sigma}^{\dagger} d_{\sigma}+U n_{\uparrow} n_{\downarrow}
$$

with a single electron of the orbital energy $\epsilon_{0}$, the Coulomb repulsion energy $U$, magnetic field $B, \sigma=1$ for a spin-up electron and $\sigma=-1$ for a spin-down electron, $d_{\sigma}^{\dagger}$ and $d_{\sigma}$ forming a set of orthogonal creation and annihilation operators for the dot and $n_{\sigma}=d_{\sigma}^{\dagger} d_{\sigma}$ being an occupation number operator. The tunneling processes between the leads and the central region are taken into account via the tunneling Hamiltonian

$$
H_{T}=\sum_{j} \sum_{k \sigma}\left(t_{j, k \sigma} c_{j, k \sigma}^{\dagger} d_{\sigma}+\text { h.c. }\right),
$$

where $t_{j, k \sigma}$ is a spin-dependent tunneling amplitude.

To include the complete magnetization of one of the leads, say lead $j$, one should limit the corresponding Hilbert space to one spin direction replacing $\sigma$ by $\uparrow$ or $\downarrow$.

\section{SEQUENTIAL TUNNELING REGIME}

The simplest situation we study is the sequential tunneling regime, also called the weak tunneling regime. It is assumed that the time between tunneling events is the largest time scale in the problem, so that there is no coherence between successive tunneling processes. If there is no bias applied, the distribution function of different states is given by the equilibrium Gibbs function. With an applied voltage difference between the electrodes the induced non-equilibrium distribution function needs to be determined.

To this end, we calculate the transition rates between different dot's states. Since we consider the weak tunneling regime, the Fermi's Golden Rule is sufficient to tackle this problem. We define the transition rates $\Gamma_{\mu \nu}^{j}$ as the rate for a process that changes the state of the dot from $\nu$ to $\mu$ due to tunneling through junction $j$. Assuming a continuous density of states inside the electrodes it follows that the tunneling rates are proportionate to the Fermi function, $\Gamma_{\nu+1, \nu}^{j}=\Gamma_{j}^{0} n\left(\epsilon_{\nu+1}-\epsilon_{\nu}-\mu_{j}\right)$, if an electron jumps onto the dot, and $\Gamma_{\nu-1, \nu}^{j}=\Gamma_{j}^{0}\left(1-n\left(\epsilon_{\nu-1}-\right.\right.$ $\left.\left.\epsilon_{\nu}-\mu_{j}\right)\right)$ for the opposite process. Here $\mu_{j}$ is a chemical potential of reservoir $j, \Gamma_{j}^{0} \equiv 2 \pi\left|t_{j}\right|^{2} \rho_{j}$ with $\rho_{i}$ being the density of states in lead $j$. To include the collinear polarizations $P_{i}$ of the leads spin-dependent tunneling rates $\Gamma_{i \sigma}^{0}=\frac{1}{2} \Gamma_{i}^{0}\left(1+\sigma P_{i}\right)$ are introduced.

Having found the transitions rates, we can write down master equations describing the dynamical behavior of the distribution function $P_{\nu}$ and since we are only interested in the steady state solution, we have 


$$
\left(\begin{array}{cccc}
-\Gamma_{\uparrow 0}-\Gamma_{\downarrow 0} & \Gamma_{0 \uparrow} & \Gamma_{0 \downarrow} & 0 \\
\Gamma_{\uparrow 0} & -\Gamma_{2 \uparrow}-\Gamma_{0 \uparrow} & 0 & \Gamma_{\uparrow 2} \\
\Gamma_{\downarrow 0} & 0 & -\Gamma_{2 \downarrow}-\Gamma_{0 \downarrow} & \Gamma_{\downarrow 2} \\
0 & \Gamma_{2 \uparrow} & \Gamma_{2 \downarrow} & -\Gamma_{\uparrow 2}-\Gamma_{\downarrow 2}
\end{array}\right)\left(\begin{array}{c}
P_{0} \\
P_{\uparrow} \\
P_{\downarrow} \\
P_{2}
\end{array}\right)=0,
$$

where $\Gamma_{\mu \nu}=\sum_{j} \Gamma_{\mu \nu}^{j}$ is a total contribution from all the leads to the transition rate from state $\nu$ to state $\mu$, while $P_{0}, P_{\uparrow}, P_{\downarrow}, P_{2}$ are the empty, spin-up, spin-down and double occupation state distribution functions respectively. The terms with a minus sign give the rate at which a given state on the left-hand side decays while the plus-sign terms describe the opposite processes. In addition to these equations, the probability conservation law $P_{0}+P_{\uparrow}+P_{\downarrow}+P_{2}=1$ has to be used.

The knowledge of the distribution functions allows one to find the net current flowing through junction $i$

$$
J_{i}=-\frac{e}{\hbar} \sum_{\nu}\left(\Gamma_{\nu+1, \nu}^{i}-\Gamma_{\nu-1, \nu}^{i}\right) P_{\nu}
$$

This should be understood as a difference between the number of electrons incoming to the dot and deoccupating it, times the electron charge, $-e<0$.

We choose the Fermi level of the stem reservoir to be our reference level, $\mu_{s}=0$, throughout the text and in all diagrams.

The solutions to Eqs. (5) are

$$
\begin{aligned}
& P_{0}=\frac{1}{D}\left(\Gamma_{0 \uparrow} \Gamma_{\uparrow 2}\left(\Gamma_{0 \downarrow}+\Gamma_{2 \downarrow}\right)+\Gamma_{0 \downarrow} \Gamma_{\downarrow 2}\left(\Gamma_{0 \uparrow}+\Gamma_{2 \uparrow}\right)\right), \\
& P_{\uparrow}=\frac{1}{D}\left(\Gamma_{\uparrow 0} \Gamma_{0 \downarrow}\left(\Gamma_{\downarrow 2}+\Gamma_{\uparrow 2}\right)+\Gamma_{\uparrow 2} \Gamma_{2 \downarrow}\left(\Gamma_{\downarrow 0}+\Gamma_{\uparrow 0}\right)\right), \\
& P_{\downarrow}=\frac{1}{D}\left(\Gamma_{\downarrow 0} \Gamma_{0 \uparrow}\left(\Gamma_{\downarrow 2}+\Gamma_{\uparrow 2}\right)+\Gamma_{\downarrow 2} \Gamma_{2 \uparrow}\left(\Gamma_{\downarrow 0}+\Gamma_{\uparrow 0}\right)\right), \\
& P_{2}=\frac{1}{D}\left(\Gamma_{2 \uparrow} \Gamma_{\uparrow 0}\left(\Gamma_{0 \downarrow}+\Gamma_{2 \downarrow}\right)+\Gamma_{2 \downarrow} \Gamma_{\downarrow 0}\left(\Gamma_{0 \uparrow}+\Gamma_{2 \uparrow}\right)\right),
\end{aligned}
$$

with

$$
\begin{aligned}
D & =\left(\Gamma_{\downarrow 0} \Gamma_{0 \uparrow}+\Gamma_{2 \uparrow} \Gamma_{\uparrow 0}\right)\left(\Gamma_{\downarrow 2}+\Gamma_{2 \downarrow}\right) \\
& +\left(\Gamma_{0 \downarrow} \Gamma_{\downarrow 2}+\Gamma_{\uparrow 2} \Gamma_{2 \downarrow}\right)\left(\Gamma_{\uparrow 0}+\Gamma_{0 \uparrow}\right) \\
& +\left(\Gamma_{0 \uparrow} \Gamma_{\uparrow 2}+\Gamma_{\downarrow 2} \Gamma_{2 \uparrow}\right)\left(\Gamma_{\downarrow 0}+\Gamma_{0 \downarrow}\right) \\
& +\left(\Gamma_{\uparrow 0} \Gamma_{0 \downarrow}+\Gamma_{2 \downarrow} \Gamma_{\downarrow 0}\right)\left(\Gamma_{\uparrow 2}+\Gamma_{2 \uparrow}\right) .
\end{aligned}
$$

Using Eq. (6) the stem current becomes

$$
\begin{aligned}
J_{s}=- & \frac{e}{\hbar}\left(\left(\Gamma_{\uparrow 0}^{s}+\Gamma_{\downarrow 0}^{s}\right) P_{0}+\left(\Gamma_{2 \uparrow}^{s}-\Gamma_{0 \uparrow}^{s}\right) P_{\uparrow}\right. \\
& \left.+\left(\Gamma_{2 \downarrow}^{s}-\Gamma_{0 \downarrow}^{s}\right) P_{\downarrow}-\left(\Gamma_{\downarrow 2}^{s}+\Gamma_{\uparrow 2}^{s}\right) P_{2}\right) .
\end{aligned}
$$

The above expression is a complicated combination of the Fermi functions and analytic treatment is not further possible. We discuss numerical results in Sec. V

\section{COTUNNELING REGIME}

As discussed in the introduction, one could expect the cotunneling current to modify the lowest order current significantly. However, this turns out not to be the case, since the cotunneling current also has nodes in the particle-hole symmetric points and only give small corrections to the overall shape of the current versus gate or voltage curves.

In the cotunneling regime two electron processes come into play. An electron is transferred between lead $j$ and lead $i$ (via an intermediate classically forbidden state) in two successive tunneling events, across the quantum dot. For the calculation of the two-electron rates, we need to consider the different starting configurations (empty, single- and double-occupied). The probabilities that a given state is occupied are given by Eqs. (77). Furthermore, there are two types of processes that should be considered, i.e. non-spin-flip processes that do not change dot's magnetization, and spin-flip processes leading to reversal of spin direction, see Fig. 3. The latter contribute directly to the current as well as modify the probabilities $P_{\sigma}$ via spin-flips caused by the interaction of the dot with the lead. We find the rates for these processes employing the generalized Fermi's Golden Rule

$$
\Gamma_{f i}=\frac{2 \pi}{\hbar}|\langle f|T| i\rangle|^{2} \delta\left(E_{f}-E_{i}\right)
$$

for the transition from the initial state $|i\rangle$ of energy $E_{i}$ to the final state $|f\rangle$ of energy $E_{f}$. The transition operator $T$ is defined as

$$
T=H_{T}+H_{T} \frac{1}{E_{i}-H_{0}} T
$$

with $H_{0}=H_{L}+H_{D}$. The first non-vanishing transition rate for the process which transfers the electron between the electrodes appears in the perturbation expansion in second order, thus the cotunneling process is quadratic in couplings, $\Gamma$.

The cotunneling events are two-particle processes and therefore occur only between pairs of the leads. Hence, the cotunneling current through junction $i$ can be expressed as the sum of the currents between any pair of leads $\tilde{J}_{i j}^{\nu}$, i.e. the currents between lead $i$ and $j$ in regime $\nu$, weighted by the appropriate probability $P_{\nu}$

$$
\tilde{J}_{i}=\sum_{j}\left(\sum_{\nu} P_{\nu} \tilde{J}_{i j}^{\nu}+\sum_{\sigma} P_{\sigma} \tilde{J}_{i j}^{\sigma, s f}\right) .
$$

The sum runs over dot's states $\nu=0, \uparrow, \downarrow, 2$, and for emphasis we divided the current into the non-spin-flip 
part $\tilde{J}_{i j}^{\nu}$ and the spin-flip part $\tilde{J}_{i j}^{\sigma, s f}$ (present for the single occupation only).

\section{A. Non-spin-flip cotunneling current}

We exemplify the derivation of the current between a pair of leads by the case of the empty dot. The initial state $|i\rangle=\left|\nu_{1}, \ldots, \nu_{N}, 0\right\rangle$ consists of a tensor product of lead's states $\left|\nu_{i}\right\rangle$ and the dot's state $|0\rangle$. The electron can be transferred from lead $i$ into lead $j$ via either a spin-up or spin-down state of the dot, depending on a spin of the electron entering the intermediate region. Because the two corresponding final states of the leads are different $|f\rangle=\left|\nu_{1}, \ldots, \nu_{i}-\sigma, \ldots, \nu_{j}+\sigma, \ldots, \nu_{N}, 0\right\rangle=c_{i, k \sigma} c_{j, k^{\prime} \sigma}^{\dagger}|i\rangle$, there is no interference between electron's paths and we find the rates $\Gamma_{j \sigma i \sigma}^{0}$ for these two processes separately and add them up to get the total tunneling rate in this regime. Thus, the current between the pair of leads $i, j$ is given by

$$
\tilde{J}_{i j}^{0}=-e \sum_{\sigma}\left(\Gamma_{j \sigma i \sigma}^{0}-\Gamma_{i \sigma j \sigma}^{0}\right)
$$

that is the rate for the process bringing the electron from lead $i$ to lead $j$ through the empty state, minus the rate for the opposite process, multiplied by the electron charge, $-e$. We substitute the tunneling Hamiltonian $H_{T}$, Eq. (4), into $\langle f|T| i\rangle$ and after a number of standard calculations, where we use (i) that the distribution function of electron states in the leads are given by the Fermi-Dirac distribution functions and (ii) the assumption of a constant tunneling density of states, we arrive at

$\Gamma_{j \sigma i \sigma}^{0}=\frac{1}{h} \Gamma_{i \sigma}^{0} \Gamma_{j \sigma}^{0} \int_{-\infty}^{\infty} d \xi \frac{1}{\left(\xi-\epsilon_{\sigma}\right)^{2}} n\left(\xi-\mu_{i}\right)\left(1-n\left(\xi-\mu_{j}\right)\right)$

Employing Eq. (13), the current between lead $i$ and $j$ through the empty dot becomes

$$
\begin{aligned}
\tilde{J}_{i j}^{0}=-\frac{e}{h} \Gamma_{i}^{0} \Gamma_{j}^{0} \int_{-\infty}^{\infty} & d \xi\left(\frac{1}{\left(\xi-\epsilon_{\uparrow}\right)^{2}}+\frac{1}{\left(\xi-\epsilon_{\downarrow}\right)^{2}}\right) \\
& \times\left(n\left(\xi-\mu_{i}\right)-n\left(\xi-\mu_{j}\right)\right),
\end{aligned}
$$

where we took $\Gamma_{i}^{0}=\Gamma_{i \uparrow}^{0}=\Gamma_{i \downarrow}^{0}$. If one neglects the inelastic processes, the currents through the single occupied dot may be found by analogy

$$
\begin{aligned}
\tilde{J}_{i j}^{\sigma}=-\frac{e}{h} \Gamma_{i}^{0} \Gamma_{j}^{0} \int_{-\infty}^{\infty} & d \xi\left(\frac{1}{\left(\xi-\epsilon_{\sigma}\right)^{2}}+\frac{1}{\left(\xi-\epsilon_{\bar{\sigma}}-U\right)^{2}}\right) \\
& \times\left(n\left(\xi-\mu_{i}\right)-n\left(\xi-\mu_{j}\right)\right)
\end{aligned}
$$

with $\bar{\sigma}=-\sigma$. The expression for the current in the presence of spin-flipping will be derived below as it needs more attention.

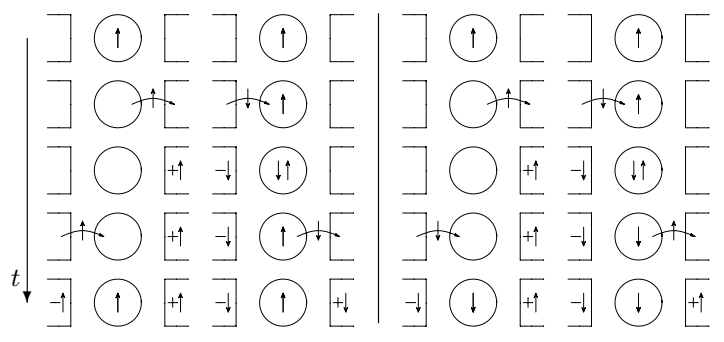

FIG. 3: The comparison of a cotunneling event for the dot occupied by a spin-up electron with the spin-flip process (on the right), and without (on the left). In case of inelastic processes, the final state of the dot and the leads is the same for both paths resulting in the interference.

Finally, the currents $J_{i j}^{2}$ in the remaining regime of the double occupation reads

$$
\begin{aligned}
\tilde{J}_{i j}^{2}=-\frac{e}{h} \Gamma_{i}^{0} \Gamma_{j}^{0} \int_{-\infty}^{\infty} & d \xi\left(\frac{1}{\left(\xi-\epsilon_{\uparrow}-U\right)^{2}}+\frac{1}{\left(\xi-\epsilon_{\downarrow}-U\right)^{2}}\right) \\
& \times\left(n\left(\xi-\mu_{i}\right)-n\left(\xi-\mu_{j}\right)\right) . \quad(15 \mathrm{c})
\end{aligned}
$$

These expressions are, however, divergent and we need to improve on the second order perturbation theory (in $\Gamma)$ to regularize these divergences. Before we follow the regularization procedure in Sec. IV C, we study the current flowing with reversal of the spin accumulated on the dot.

\section{B. Spin-flip cotunneling current}

As long as we consider the limit of non-interacting electrons, $U=0$, the inelastic processes do not affect the current at all, and the above description is completely sufficient. In the Appendix we compare the perturbative result with the exact results obtainable when neglecting interactions.

The derivation of the tunneling rate and the current between a pair of leads in the presence of spin-flip processes is similar to one used in the previous section. Here, we just cite the result

$$
\tilde{J}_{i j}^{\sigma, s f}=-e\left(\Gamma_{j \bar{\sigma} i \sigma}-\Gamma_{i \bar{\sigma} j \sigma}\right)
$$

with the tunneling rate

$$
\begin{aligned}
\Gamma_{j \bar{\sigma} i \sigma}=\frac{1}{h} \Gamma_{i \sigma}^{0} \Gamma_{j \bar{\sigma}}^{0} \int_{-\infty}^{\infty} & d \xi\left(\frac{1}{\xi-\epsilon_{\bar{\sigma}}-U}-\frac{1}{\xi-\epsilon_{\bar{\sigma}}}\right)^{2} \\
& \times n\left(\xi-\mu_{i}\right)\left(1-n\left(\xi-\mu_{j}-\Delta_{\bar{\sigma} \sigma}\right)\right) .
\end{aligned}
$$

This expression differs from that for the cotunneling current without spin-flip processes, Eq. (15b), as the interference term appears there, Fig. 3. Furthermore, the chemical potential of lead $j$ is shifted due to the energy gap, $\Delta_{\bar{\sigma} \sigma} \equiv \epsilon_{\bar{\sigma}}-\epsilon_{\sigma}$, resulting from the Zeeman splitting. It is convenient to rewrite the expression 
$n\left(\xi-\mu_{i}\right)\left(1-n\left(\xi-\mu_{j}+\Delta_{\bar{\sigma} \sigma}\right)\right)=n_{B}\left(\mu_{j}-\mu_{i}+\Delta_{\bar{\sigma} \sigma}\right) \times$ $\left(n\left(\xi-\mu_{j}-\Delta_{\bar{\sigma} \sigma}\right)-n\left(\xi-\mu_{i}\right)\right)$ so that it has the same form as Eqs. (15), i.e. it is proportional to the Fermi functions' difference, with $n_{B}$ standing for the Boltzmann function. In the next section it will come to light that terms of this type undergo the regularization scheme.

\section{Regularization procedure}

In general for the non-spin-flip cotunneling current, the problematic integrand is a product of the divergent term $\left(\xi-\xi_{0}\right)^{-2}$ and the Fermi functions' difference, denoted as $f(\xi) \equiv n\left(\xi-\mu_{i}\right)-n\left(\xi-\mu_{j}\right)$.

To deal with this divergence we follow the regularization scheme proposed by Turek and Matveev ${ }^{22,23}$ and add to the denominator a life-time broadening, $\eta^{2}$, describing the tunneling broadening of the intermediate state, i.e. $\eta \propto \Gamma$. The whole trick is to partition this integral into two parts, from which the first can be a posteriori identified as the energy conserving process, and hence, the sequential tunneling contribution while the second term describes the regularized cotunneling processes. This is done as

$$
\begin{aligned}
\int d \xi & \frac{f(\xi)}{\left(\xi-\xi_{0}\right)^{2}+\eta^{2}} \\
& =\int d \xi \frac{f\left(\xi_{0}\right)}{\left(\xi-\xi_{0}\right)^{2}+\eta^{2}}+\int d \xi \frac{f(\xi)-f\left(\xi_{0}\right)}{\left(\xi-\xi_{0}\right)^{2}+\eta^{2}} \\
& \longrightarrow \frac{\pi}{|\eta|} f\left(\xi_{0}\right)+\lim _{\eta \rightarrow 0^{+}} \int d \xi \frac{f(\xi)-f\left(\xi_{0}\right)}{\left(\xi-\xi_{0}\right)^{2}+\eta^{2}}
\end{aligned}
$$

where the last line is in the limit of small $\eta$. The first term corresponds to the transitions on resonance (or "on-shell"), where energy is conserved in each tunneling event. These processes give rise to a current which is linear in $\Gamma$ after inserting into Eq. (12) and using that $\eta \propto \Gamma$. We can therefore omit the first term, since it has already been calculated within the much simpler master equation scheme in Sec. [II] The remaining term now corresponds to the proper regularized second order contribution to the current. At this point it is important to realize that this procedure does not capture all second order terms, because the renormalization of the dot state due to tunneling is not included $\stackrel{1}{=}$ The effect of renormalization, however, is easily incorporated by adding these terms (linear in $\Gamma$ ) to the energies of the dot states when doing the master equations. The second order correction (in $\Gamma$ ) can then be extracted from the master equation result. Here we do not incorporated them since they are unimportant and merely give a shift of the gate voltage. On the other hand, for some cases, e.g. non-collinear magnetization, they cannot be neglected because they give rise to off-diagonal elements in the density matrix. ${ }^{7}$

Now returning to the expression (18) we can evaluate it analytically by employing the useful identity

$$
\lim _{\eta \rightarrow 0^{+}} \int d \xi \frac{f(\xi)-f\left(\xi_{0}\right)}{\left(\xi-\xi_{0}\right)^{2}+\eta^{2}}=\lim _{\eta \rightarrow 0^{+}} \frac{\partial}{\partial \xi_{0}} \operatorname{Re} \int d \xi \frac{f(\xi)}{\xi-\xi_{0}+i \eta}
$$

and summing over the residues. The final result becomes

$$
\begin{aligned}
\int_{-\infty}^{\infty} & \frac{d \xi}{\left(\xi-\xi_{0}\right)^{2}}\left(n\left(\xi-\mu_{i}\right)-n\left(\xi-\mu_{j}\right)\right) \\
& \longrightarrow \operatorname{Re}\left[\frac{\beta}{2 \pi i}\left(\Psi_{1}\left(\xi_{0}, \mu_{i}\right)-\Psi_{1}\left(\xi_{0}, \mu_{j}\right)\right)\right] .
\end{aligned}
$$

The arrow indicates that the divergent integral has been regularized by the procedure explained above. For convenience we introduced a shorthand notation for the polygamma function of $\mathrm{n}$ - $t h$ order

$$
\Psi_{n}(\xi, \mu) \equiv \Psi_{n}\left(\frac{1}{2}-\frac{\beta}{2 \pi i}(\xi-\mu)\right) .
$$

Below we list the non-spin-flip currents, Eqs. (15) after the regularization (for more detailed derivation of regularized formulas see Ref. 24)

$$
\begin{gathered}
\tilde{J}_{i j}^{0}=-\frac{e}{h} \Gamma_{i}^{0} \Gamma_{j}^{0} \operatorname{Re}\left[\frac{\beta}{2 \pi i}\left(\Psi_{1}\left(\epsilon_{\uparrow}, \mu_{i}\right)-\Psi_{1}\left(\epsilon_{\uparrow}, \mu_{j}\right)\right)\right. \\
\left.+\frac{\beta}{2 \pi i}\left(\Psi_{1}\left(\epsilon_{\downarrow}, \mu_{i}\right)-\Psi_{1}\left(\epsilon_{\downarrow}, \mu_{j}\right)\right)\right], \quad(22 \mathrm{a}) \\
\tilde{J}_{i j}^{\sigma}=-\frac{e}{h} \Gamma_{i}^{0} \Gamma_{j}^{0} \operatorname{Re}\left[\frac{\beta}{2 \pi i}\left(\Psi_{1}\left(\epsilon_{\sigma}, \mu_{i}\right)-\Psi_{1}\left(\epsilon_{\sigma}, \mu_{j}\right)\right)\right. \\
\left.+\frac{\beta}{2 \pi i}\left(\Psi_{1}\left(\epsilon_{\bar{\sigma}}+U, \mu_{i}\right)-\Psi_{1}\left(\epsilon_{\bar{\sigma}}+U, \mu_{j}\right)\right)\right], \quad(22 \mathrm{~b}) \\
\tilde{J}_{i j}^{2}=-\frac{e}{h} \Gamma_{i}^{0} \Gamma_{j}^{0} \operatorname{Re}\left[\frac{\beta}{2 \pi i}\left(\Psi_{1}\left(\epsilon_{\uparrow}+U, \mu_{i}\right)-\Psi_{1}\left(\epsilon_{\uparrow}+U, \mu_{j}\right)\right)\right. \\
\left.+\frac{\beta}{2 \pi i}\left(\Psi_{1}\left(\epsilon_{\downarrow}+U, \mu_{i}\right)-\Psi_{1}\left(\epsilon_{\downarrow}+U, \mu_{j}\right)\right)\right] . \quad(22 \mathrm{c})
\end{gathered}
$$

The current in the presence of the inelastic scattering, Eq. (16), causes more problems as the interference term emerges. Using the partial fraction decomposition it turns to be

$$
\begin{aligned}
& \left(\frac{1}{\xi-\xi_{1}}-\frac{1}{\xi-\xi_{2}}\right)^{2}= \\
& \quad=\frac{1}{\left(\xi-\xi_{1}\right)^{2}}+\frac{1}{\left(\xi-\xi_{2}\right)^{2}}-\frac{2}{\xi_{1}-\xi_{2}}\left(\frac{1}{\xi-\xi_{1}}-\frac{1}{\xi-\xi_{2}}\right)
\end{aligned}
$$

with the new type of divergence $\left(\xi-\xi_{0}\right)^{-1}$. The identity

$$
\lim _{\eta \rightarrow 0^{+}} \int d \xi \frac{g(\xi)-g\left(\xi_{1}\right)}{\left(\xi-\xi_{1}\right)^{2}+\eta^{2}}=\lim _{\eta \rightarrow 0^{+}} \operatorname{Re} \int d \xi \frac{f(\xi)}{\xi-\xi_{1}+i \eta},
$$

where $g(\xi) \equiv\left(\xi-\xi_{1}+\frac{2 \eta^{2}}{\xi_{1}-\xi_{2}}\right) f(\xi)$, allows one to find the regularized expression for this type of divergence as well

$$
\begin{aligned}
& \int_{-\infty}^{\infty} \frac{d \xi}{\xi-\xi_{0}}\left(n\left(\xi-\mu_{i}\right)-n\left(\xi-\mu_{j}\right)\right) \\
& \quad \longrightarrow \operatorname{Re}\left[\Psi_{0}\left(\left(\xi_{0}, \mu_{j}\right)-\Psi_{0}\left(\xi_{0}, \mu_{i}\right)\right)\right]
\end{aligned}
$$



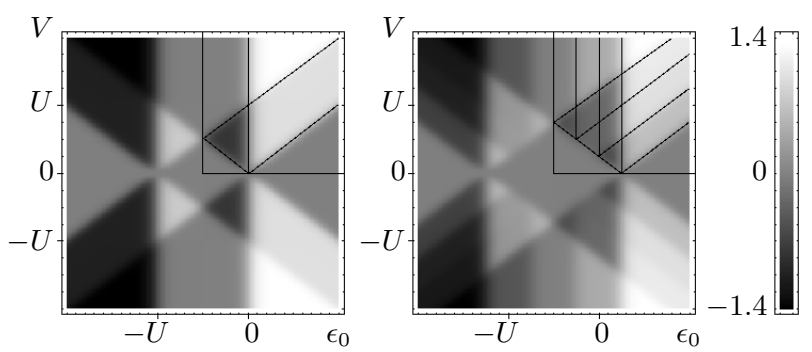

FIG. 4: The stem current in function of the bias voltage $V$ and the gate voltage $\epsilon_{0}$. The leads are unpolarized and equally coupled to the dot, with coupling's strength $\Gamma / 3$. The picture without the magnetic field (on the left) differs from this with field $B=5 \Gamma$ (on the right). The interaction energy $U=20 \Gamma$ and temperature $k_{B} T=\Gamma$. The grey scale in the far right describes the stem current value in units $e \Gamma / \hbar$.

and after some algebra the inelastic tunneling rates become

$$
\begin{aligned}
\Gamma_{j \bar{\sigma} i \sigma}= & \frac{1}{h} \Gamma_{i \sigma}^{0} \Gamma_{j \bar{\sigma}}^{0} n_{B}\left(\mu_{j}+\Delta_{\bar{\sigma} \sigma}-\mu_{i}\right) \\
\times & \operatorname{Re}\left[\frac{\beta}{2 \pi i}\left(\Psi_{1}\left(\epsilon_{\bar{\sigma}}+U, \mu_{j}+\Delta_{\bar{\sigma} \sigma}\right)-\Psi_{1}\left(\epsilon_{\bar{\sigma}}+U, \mu_{i}\right)\right)\right. \\
& +\frac{\beta}{2 \pi i}\left(\Psi_{1}\left(\epsilon_{\bar{\sigma}}, \mu_{j}+\Delta_{\bar{\sigma} \sigma}\right)-\Psi_{1}\left(\epsilon_{\bar{\sigma}}, \mu_{i}\right)\right) \\
& +\frac{2}{U}\left(\Psi_{0}\left(\epsilon_{\bar{\sigma}}+U, \mu_{j}+\Delta_{\bar{\sigma} \sigma}\right)-\Psi_{0}\left(\epsilon_{\bar{\sigma}}+U, \mu_{i}\right)\right) \\
& \left.-\frac{2}{U}\left(\Psi_{0}\left(\epsilon_{\bar{\sigma}}, \mu_{j}+\Delta_{\bar{\sigma} \sigma}\right)-\Psi_{0}\left(\epsilon_{\bar{\sigma}}, \mu_{i}\right)\right)\right]
\end{aligned}
$$

which substituted to Eq. (16) gives a proper limit of noninteracting electrons, $\lim _{U \rightarrow 0} \tilde{J}_{i j}^{\sigma, s f}=0$. Note, that current between each set of leads (involving spin's reversal or not) fulfils the principle of detailed balance (vanishes for $\mu_{i}=\mu_{j}$ ) and, hence, the total cotunneling current is equal to zero, when the chemical potentials of the leads are at the same level.

\section{RESULTS}

In Fig. 4 the stem current dependence upon the bias voltage $V$ and the gate voltage $\epsilon_{0}$ for no magnetic field (the left plot) and for magnetic field $B=5 \Gamma$ (right) is shown. Bright regions correspond to positive values of the current while dark areas to negative ones. It is apparent that the stem current is an even function of bias $V$ and an odd function of the orbital energy $\epsilon_{0}$ with respect to the particle-hole symmetry line $\epsilon_{0}=-U / 2$. Therefore, we restrict our discussion to the parts of the plots where $V>0$ and $\epsilon_{0}>-U / 2$. By performing the particle-hole transformation, the behavior in region where $\epsilon_{0}>-U / 2$ holds, can be mapped onto the remaining area of the $V-\epsilon_{0}$ space.

Consider the case without the magnetic field first. If $\epsilon_{0}<0$, the dot is in the single occupied state, and until $V<\epsilon_{0}$ the transport is blocked because electrons

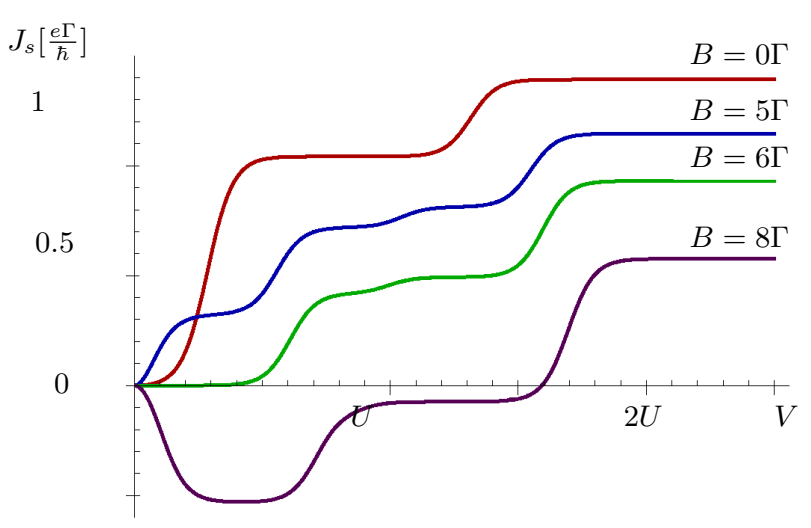

FIG. 5: (Color online) The stem current for different values of magnetic field $B$ is plotted. Interestingly, there is a sign change in the vicinity of $V=0$ for $B=\epsilon_{0}$. See text for explanation. The gate voltage is $\epsilon_{0}=6 \Gamma$. Other parameters are as in the previous figure.

from the left branch do not have enough energy to overcome the Coulomb blockade. Increasing voltage makes the transport out of the stem possible, since electrons may escape to the empty states in the right lead. However, further increase of the voltage stops the stem current again, because for $V>\epsilon_{0}+U$ (when two electrons excitations become possible) the left reservoir supplies the stem with electrons while at the same time the electrons from the stem move into the right reservoir. These currents cancel one another and there is no net stem current. The situation differs for $\epsilon_{0}>0$. The current increases once $V>\epsilon_{0}$, when dot is excited to the single occupied state, and again when $V>\epsilon_{0}+U$, where the electrons transverse through the double occupied state. In both cases the direction of the flux is directed into the stem and carried by the left-lead electrons. Clearly, for the negative voltages the role of the right and left reservoir interchanges, but nevertheless the direction of the stem current is unaffected.

In the presence of the magnetic field more complex structure emerges due to two new excitations coming into play (as the single occupied state is no longer degenerate). It is convenient to divide the right part of the diagram into four vertical strips $\epsilon_{0} \in[-U / 2,-U / 2+B]$, $[-U / 2+B, 0],[0, B]$ and $\epsilon_{0}>B$.

In the first region, the negative stem current flows in the narrow range of voltages. Below the lower threshold $V<-\epsilon_{0}+B$, the spin-up electron occupying the dot stops the current until the required state in the right reservoir becomes available. Then, both spin-up and spin-down electrons can participate in transport. For $V>\epsilon_{0}+U+B$ the electrons from the left branch enter and compensate the current carried from the stem to the right lead and, hence, the net stem current vanishes.

In the second region, from $V=0$ to $V=-\epsilon_{0}+B$ the dot is occupied by the spin-up electron which blocks the current. Beyond $V=-\epsilon_{0}+B$ the spin-up states in the right lead become available and this electron moves 

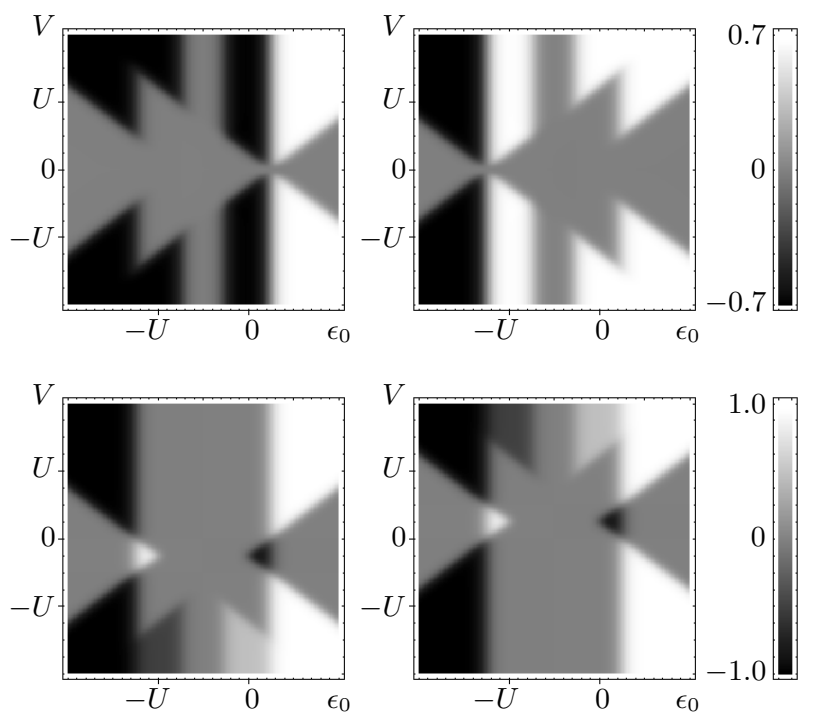

FIG. 6: The stem current for different polarizations of leads and magnetic field $B=5 \Gamma$. The central branch is always unpolarized. In the upper row, both left and right leads are spin-up polarized, $P_{l}=P_{r}=1$ (the left plot), or spin-down polarized, $P_{l}=P_{r}=-1$ (the right plot). Due to nonzero magnetic field, the spin symmetry is broken and the stem current is no longer an odd function of the gate voltage with respect to $\epsilon_{0}=-U / 2$. In the lower row, polarization of the left and right leads is antiparallel $\left(P_{l}=1, P_{r}=-1\right.$ on the left, and $P_{l}=-1, P_{r}=1$ on the right). In this case, the current symmetry in $V$ is violated, because the dot couples to the left and right leads asymmetrically. The other parameters are as in the previous figure.

towards the right reservoir. Further increase of the bias voltage results in a small increase of the current at $V=$ $\epsilon_{0}+U-B$, when spin-up electrons from the left electrode can pass through the Coulomb blockade. Finally, there is a sign change of the stem current at $V>\epsilon_{0}+U+B$, because electrons of both spin directions go into the stem from the left lead while still only spin-up electrons can move into the right reservoir.

The negative stem current in the third strip starts to flow when $V>-\epsilon_{0}+B$ due to the spin-up electrons moving out of the stem into the right reservoir. This current is risen by the spin-down electrons from the left lead at $V=\epsilon_{0}+B$ and also by electrons moving through the double occupied state (for both $V=\epsilon_{0}+U-B$ and $\left.V=\epsilon_{0}+U+B\right)$.

Eventually, in the last strip the stem current has four steps. These are for $V=\epsilon_{0}-B, V=\epsilon_{0}+B$ when the excitations of the empty dot to spin-up and spin-down state respectively become energetically allowed, and for $V=\epsilon_{0}+U-B, V=\epsilon_{0}+U+B$ when electrons from the right lead have enough energy to overcome the Coulomb blockade (due to the spin-down and then spin-up electron on the dot).

Fig. 5 shows how the current is affected by the magnetic field applied to the dot. The stem current remains positive as long as $B \leq \epsilon_{0}$ for any bias. The character of the function changes when $B$ crosses $\epsilon_{0}$. This corresponds to the situation when the single occupancy state has the same energy as the empty state (the electron-hole symmetry point). Tuning the magnetic field to $B=\epsilon_{0}$ enables one to measure directly electron-hole transport fluctuations.

Providing $B>\epsilon_{0}$, the spin-up state of the dot is below the Fermi level of the stem and for small voltages the negative current is most likely to occur. However, the increasing voltage forces more and more electrons to move out of the left lead into the central branch. These electrons overbalance those heading in the opposite direction and the current sign changes - it is apparent for $B=8 \Gamma$.

In two-lead systems the tunneling magnetoresistance (TMR) have widely been studied. ${ }^{4,5,6}$ Defining a similar quantity in the multiprobe setup is rather vague. Nonetheless, it turns out that the current through the central, unpolarized lead has interesting features while varying magnetization of the other electrodes. The results are shown in Fig. 6.

The parallel magnetization (the upper part) destroys the antisymmetry in the gate voltage whereas the antiparallel alignment (the lower part) breaks the bias voltage symmetry. It is worth to note that the third lead gives not only information about the relative magnetization of two remaining electrodes, but also allows one to determine polarization of each lead. This means that in that kind of a device we can switch among four different states and directly read out the information decoded in the two-lead system with the third probe. Experimentally it might be more convenient to swap the magnetic field on the dot rather than to change polarization of the side leads.

\section{CONCLUSIONS}

In conclusion, we have studied Coulomb blockade in a three terminal device. We have focused on the bias situation where two leads have opposite voltage, while the third lead (the stem) is grounded. This setup allows for a direct measure of the electron-hole asymmetry of the quantum dot system and we have made detailed calculations of the stem current in the gate voltage-bias voltage plane. We have pointed to a number of predictions, that can be experimentally tested.

Furthermore, we have considered the spin polarized case, where a number of detailed experimental proposals have been presented. In particular, a large difference in predicted pattern between parallel and anti-parallel configurations is seen.

Finally, we have checked, using a regularized version of the usual cotunneling formalism, that our predictions are not significantly altered by cotunneling corrections. 


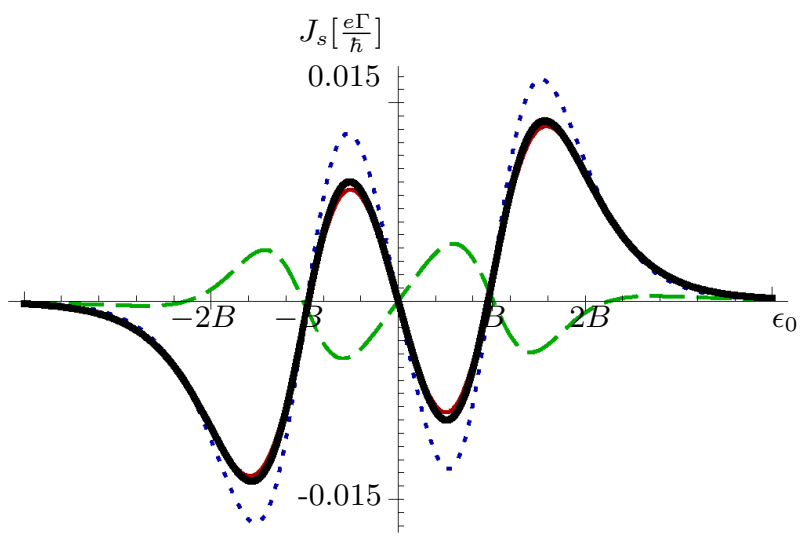

FIG. 7: (Color online) The strict result for the stem current (thick, black) compared to the perturbative result: sequential (dotted, blue), cotunneling (dashed, green), and their sum (thin, red). Parameters are as in Fig. (2) (except for $U=0$ ).

\section{APPENDIX: NON-INTERACTING ELECTRONS LIMIT}

The limiting case of non-interacting electrons gives the opportunity to compare the results we get within the framework of the second order perturbation theory with the exact result containing higher order terms in couplings. The quality of the cut-off on the second order terms can be estimated. We derive the generalization of the Meir-Wingreen formula that will include the indefinite number of leads and calculate the current $J_{i}$ through one of the junctions.

The main result of Meir and Wingreen 25 is the current through lead $i$ in the presence of interactions

$J_{i}=\frac{i e}{h} \int d \xi\left\{\operatorname{Tr}\left[\Gamma_{i}\left(G^{R}-G^{A}\right)\right] n\left(\xi-\mu_{i}\right)+\operatorname{Tr}\left[\Gamma_{i} G^{<}\right]\right\}$.

This can be generalized to the many lead systems employing the obvious identity $J_{i}=\gamma J_{i}-(1-\gamma) \sum_{j \neq i} J_{j}$ following from Kirchhoff's law. Providing all the couplings are proportionate, that is $\Gamma_{i}=\lambda_{i j} \Gamma_{j}$ where $\lambda_{i j}$ are constants, one may eliminate the lesser Green function $G^{<}$by proper selection of $\gamma$ showing that

$$
J_{i}=-\frac{e}{h} \int d \xi \sum_{j=1}^{N} \operatorname{Tr}\left[A \frac{\Gamma_{i} \Gamma_{j}}{\Gamma}\right]\left(n\left(\xi-\mu_{i}\right)-n\left(\xi-\mu_{j}\right)\right),
$$

where we introduced a shorthand notation $\Gamma \equiv \sum_{i} \Gamma_{i}$ and wrote the formula for the current $J_{i}$ in terms of a spectral function $A \equiv i\left(G^{R}-G^{A}\right)$.

For non-interacting electrons

$$
A(\xi, \sigma)=\frac{\Gamma}{\left(\xi-\epsilon_{\sigma}\right)^{2}+\left(\frac{\Gamma}{2}\right)^{2}}
$$

describes a spin dependent spectral function, which substituted to the generalized Meir-Wingreen formula gives the exact current in lead $i$ for the multiterminal device, valid to any order in couplings

$$
J_{i}=-\frac{e}{h} \Gamma_{i} \sum_{j} \sum_{\sigma} \Gamma_{j} \int d \xi \frac{n\left(\xi-\mu_{i}\right)-n\left(\xi-\mu_{j}\right)}{\left(\xi-\epsilon_{\sigma}\right)^{2}+\left(\frac{\Gamma}{2}\right)^{2}}
$$

We note in passing that the same result comes from the non-interacting Landauer-Bütticker formalism for any number of electrodes.

Using the similar methods as described in Sec. IV C, we derive the current through junction $i$ that depends on coupling $\Gamma$ up to any order

$$
J_{i}=-\frac{4 e}{h} \Gamma_{i} \sum_{j} \sum_{\sigma} \Gamma_{j} \operatorname{Im}\left[\Psi_{0}\left(\epsilon_{\sigma}, \mu_{i}, \Gamma\right)-\Psi_{0}\left(\epsilon_{\sigma}, \mu_{j}, \Gamma\right)\right]
$$

and

$$
\Psi_{0}\left(\epsilon_{0}, \mu, \Gamma\right) \equiv \Psi_{0}\left(\frac{1}{2}-\frac{\beta}{2 \pi i}\left(\epsilon_{0}-\mu\right)+\frac{\beta}{4 \pi} \Gamma\right) .
$$

In Fig. 7 the exact result is compared with the perturbative one. The good agreement between these two justifies the choice of the method, and reassures that the second order perturbation theory gives not only excellent quantitative description, reachable also within the sequential tunneling framework, but also good qualitative estimation, at least for non-interacting electrons. Note, however, that this conclusion is only valid in the regime where $\Gamma \ll k_{B} T$, whereas in the opposite limit the perturbation expansion clearly fails, and one does not expect the good agreement.

\section{ACKNOWLEDGMENTS}

We acknowledge inspiring discussions with A. M. Lunde, J. A. Majewski, J. Paaske, M. Wegewijs. R. A. Żak appreciates the hospitality of the Niels Bohr Institute, University of Copenhagen, and the financial support from the European Physical Society.
1 J. König, J. Schmid, H. Schoeller, and G. Schön, Phys. Rev. B 54, 16820 (1996).

2 J. Lehmann and D. Loss, Phys. Rev. B 73, 45328 (2006).

3 J. Koch, M. E. Raikh, and F. von Oppen, Phys. Rev. Lett.
96, 56803 (2006).

4 B. R. Bułka, Phys. Rev. B 62, 1186 (2000).

5 W. Rudziński and J. Barnaś, Phys. Rev. B 64, 085318 (2001). 
${ }^{6}$ I. Weymann et al., Phys. Rev. B 72, 115334 (2005).

7 J. König and J. Martinek, Phys. Rev. Lett. 90, 166602 (2003).

8 M. Braun, J. König, and J. Martinek, Phys. Rev. B 70, 195345 (2004).

9 J. Pedersen, J. Thomassen, and K. Flensberg, Phys. Rev. B 72, 45341 (2005).

10 M. Büttiker, Y. Imry, R. Landauer, and S. Pinhas, Phys. Rev. B 31, 6207 (1985).

11 T. Palm and L. Thylén, Appl. Phys. Lett. 60, 237 (1992).

12 J.-O. J. Wesström, Phys. Rev. Lett. 82, 2564 (1999).

13 K. Hieke and M. Ulfward, Phys. Rev. B 62, 16727 (2000).

14 L. Worschech, D. Hartmann, S. Reitzenstein, and A. Forchel, J. Phys.:Condens. Matter 17, 775 (2005).

15 A. Cottet, W. Belzig, and C. Bruder, Phys. Rev. B 70, 115315 (2004).

16 A. Cottet, W. Belzig, and C. Bruder, Phys. Rev. Lett. 92,
206801 (2004)

17 Q.-f. Sun and H. Guo, Phys. Rev. B 64, 153306 (2001).

18 D. Sánchez and R. López, Phys. Rev. B 71, 035315 (2005).

19 S. Chen, B. Trauzettel, and R. Egger, Phys. Rev. Lett. 89, 226404 (2002).

${ }^{20}$ R. Egger, B. Truzettel, S. Chen, and F. Siano, New J. Phys. 117, 1 (2003).

21 B. Gao et al., Phys. Rev. Lett. 92, 216804 (2004).

22 M. Turek and K. A. Matveev, Phys. Rev. B 65, 115332 (2002).

23 J. Koch, F. von Oppen, and A. V. Andreev, Phys. Rev. B 74, 205438 (2006)

${ }^{24}$ R. A. Żak, Master's thesis, University of Warsaw, 2007.

25 Y. Meir and N. Wingreen, Phys. Rev. Lett. 68, 2512 (1992). 